\title{
Which Medium and Ingredients Provide Better Morphological Differentiation of SH-SY5Y Cells? ${ }^{\dagger}$
}

\author{
Belgin Sert Serdar ${ }^{1}$, Tuğba Erkmen ${ }^{1}$, Bekir Uğur Ergür ${ }^{2}$, Pınar Akan ${ }^{1}$ and Semra Koçtürk ${ }^{1 *}$ \\ 1 Department of Biochemistry, Faculty of Medicine, Dokuz Eylul University, 35340 Izmir, Turkey; \\ belginsrt@gmail.com (B.S.S.); erkmenntuba@gmail.com (T.E.); pinar.akan@deu.edu.tr (P.A.) \\ 2 Basic Medical Sciences Histology and Embryology, Faculty of Medicine, Dokuz Eylul University, 35340 \\ Izmir, Turkey; bekir.ergur@deu.edu.tr \\ * Correspondence: semra.kocturk@deu.edu.tr \\ † Presented at the 2nd International Cell Death Research Congress, İzmir, Turkey, 1-4 of November 2018.
}

Published: 5 December 2018

\begin{abstract}
Human SH-SY5Y cell line has been used as an in vitro model in neuroscience research. However, many researchers emphasized that there are many differences between differentiated and undifferentiated characteristics of SH-SY5Y. Although Retinoic Acid (RA) generally have been used for differentiation of SH-SY5Y cells, which protocol provides better differentiation have not been cleared yet. Therefore, we compared RA and the other mediums in different treatment periods for obtaining better differentiated, carrying a neuron-like phenotype (N-type) characteristics, of SHSY5Y human cells. The cells were pre-treated with different mediums for different treatment periods and compared with both for each other and the control group. When we pre-treated the cells with RA for 5-day and followed by the Mix medium (Neurobasal, B27, db-cAMP, KCI) and BDNF, neurite length and MAP2 expression of the cells were found significantly higher than the control group. In conclusion, we showed that more than one agent (RA) is clearly necessary to reach better differentiation of SH-SY5Y cells.
\end{abstract}

Keywords: SH-SY5Y cell; neuronal differentiation; Retinoic acid; BNDF; MAP2

\section{Introduction}

Human SH-SY5Y neuroblast-like cells, a subclone of parental SK-N-SH cell line, has been used frequently in-vitro experiments in the fields of the neurosciences [1]. The cell line contains both adherent and floating cells. Research revealed that SK-N-SH cells also consist of neuroblast-like cells (N-type) and epithelial-like cells (S-type) [2]. Therefore, several studies indicated that the important difference between differentiated and undifferentiated states of SH-SY5Y cells that affecting polarization and proliferation. While undifferentiated SH-SY5Y cells can able to proliferate rapidly, grow in clumps and non-polarized, differentiated cells show decreased proliferation rate, extended neurites and more pyramidal shaped. On the other hand, differentiated SH-SY5Y cells have some biochemical characteristics and express neuronal markers such as neurofilaments heavy/light polypeptide, neuron-specific enolase (NSE), growth-associated protein-43 (GAP-43), neuronal nuclei (NeuN), tubulin $\beta$-III ( $\beta$-III Tubulin) and microtubule-associated protein 2 (MAP-2) [3-5]. Frequently used reagents in the differentiation of SH-SY5Y cells, such as Retinoic acid (RA), have been still controversial in terms of which reagent provides better differentiation to neuron-like phenotype.

For instance, in addition to RA, vitamin D3, phorbol esters, brain-derived neurotrophic factor (BDNF), nerve growth factor (NGF), dibutyryl-cyclic AMP (db-cAMP), cholesterol, estradiol, 12-Otetradecanoyl-phorbol-13-acetate are agents that can be used separately or in a combinational form for differentiation of the cells [5-7]. There is the divergence on the agents that provide better differentiation, although researchers mostly use RA, its treatment period is also variable. In literature, 
although the used concentration of RA is similar such as $10 \mu \mathrm{M}$, treatment periods show variability for $3-5$ days or $>10$ days in low serum or serum-free medium [2,5]. Encinas et al. have strongly emphasized the importance of RA treatment period on differentiation. They have argued that shortterm treatment (up to 5 days) has a capacity to induce N-type cells differentiation but longer period, such as $>10$ days leads to promote proliferation of S-type cells [2]. Researchers also emphasized that the combination of RA and BNDF has a beneficial effect on the expression of neuronal markers. Therefore, researchers have been focused on the importance of the ingredients of the mediums on the differentiation of SH-SY5Y cells. The aim of this study was to examine the effects of medium ingredients and the treatment periods on differentiation of SH-SY5Y cells having neuron-like phenotype characteristics.

\section{Materials and Methods}

Human neuroblastoma cell line SH-SY5Y (ATCC-2266) was purchased from ATCC. Cells were cultured in DMEM: F12 supplemented with FBS, penicillin, streptomycin in humidified $5 \% \mathrm{CO}_{2}$ incubator at $37^{\circ} \mathrm{C}$. We composed six groups; Group 1: control undifferentiated SH-SY5Y cells, Group 2: treated with RA for 5-day, Group 3: treated with RA for 10-day, Group 4: pre-treated with RA for 5-day followed with BDNF for 5-day, Group 5: pre-treated with RA for 5-day followed with mix medium (Neurobasal, B27, dc-AMP, KCI) for 5-day, Group 6: pre-treated with RA for 5-day followed mix medium (Neurobasal, B27, db-cAMP, KCI) and BDNF for 5-day. Cellular morphology of differentiated-SH-SY5Y cells were examined with an Olympus CKX40 digital camera. After 10 days of differentiation, the cells were fixed with $4 \%$ paraformaldehyde in a PBS solution for $30 \mathrm{~min}$ at room temperature. Permeabilized with $0.2 \%$ Triton X-100 in PBS and then incubated in blocking solution at room temperature. The cells were incubated at $37^{\circ} \mathrm{C}$ with primary antibodies (MAP2) after washes in PBS, the cells were incubated with secondary antibody at room temperature and immediately analyzed with a Leica DMIL fluorescence microscope. Neurite length was measured with the Neuron $\mathrm{J}$ analysis software. On the tenth day, the cultured cells were observed using a phase-contrast microscope, and six different locations were photographed in each well. This software is the number of neurites developed per cell was manually counted [6].

\section{Results and Discussion}

Morphological changing of differentiated and undifferentiated SH-SY5Y cells were visually analyzed at 10th days by phase contrast microscopy. Our results showed that undifferentiated SHSY5Y cells tend to grow in clusters and had no particular neurites (Figure 1a), whereas neurites have been tending to extend in the other groups. When cells pre-treated with RA for 5 days and followed with BDNF included-Mix medium (Glutamax I, Neurobasal, KCI, dc-AMP) for 5 days, the branching rate and connections of neurites were higher than the other groups (Figure 1f). Our experimental data imply that used mediums, ingredients and treatment periods have an effect and determine the differentiation level of SH-SY5Y cells. Our results that confirm the other reports in which morphology of differentiated and undifferentiated SH-SY5Y cells [5,6].

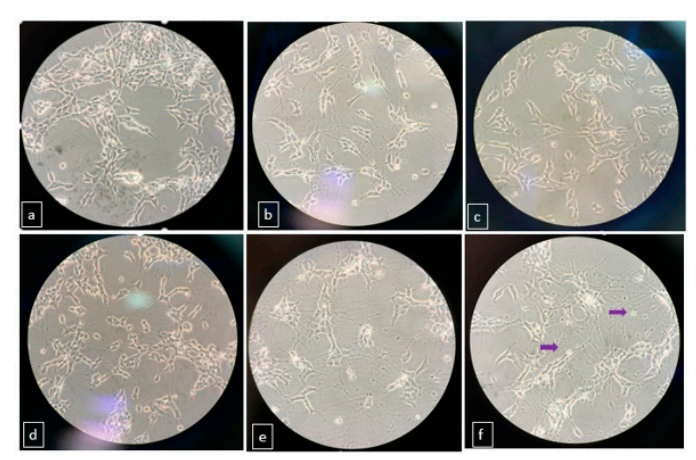

Figure 1. Morphology and network formation of differentiated and undifferentiated SH-SY5Y cells at different treatment periods. (a) undifferentiated (b) treated with RA for 5 days (c) treated with RA for 
10 days (d) pre-treated RA for 5 days then with BDNF for 5 days (e) pre-treated with RA for 5 days then with mix medium for 5 days (f) pre-treated with RA for 5 days then with mix medium and BDNF for 5 days.

As a neuronal marker, MAP2 expression was assessed by immunofluorescence assay in differentiated SH-SY5Y cells. The intensity of MAP2 fluorescence in group 6 was significantly increased in comparison to the control and the other groups (Figure 2). Our MAP2 results were compatible with the study of Mackenzie et.al [5].
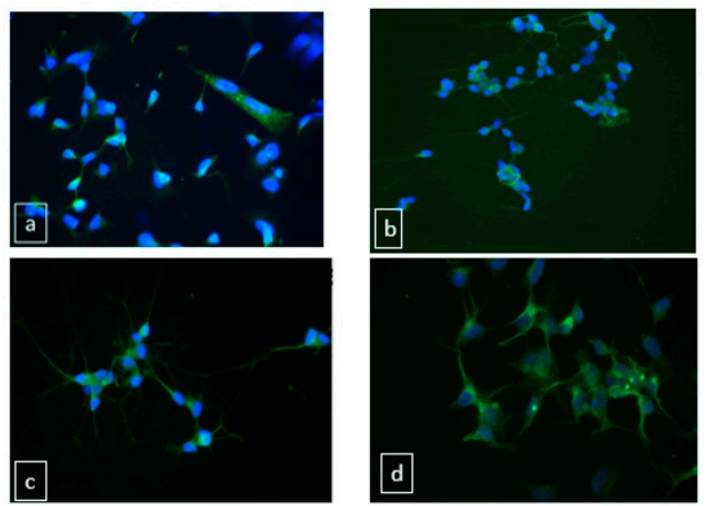

Figure 2. MAP-2 expression in differentiated and undifferentiated SH-SY5Y cells. (a) undifferentiated cells (b) pre-treated RA followed with BDNF (c) pre-treated RA followed with Mix medium (d) pretreated RA followed with Mix medium and BDNF.

The neurites length of differentiated SH-SY5Y cells were calculated by phase contrast images that were taken at 10th day. Results showed that group 6 and 5 have cells with the longest neurites than the other groups significantly $(p<0.001)$. When we compared the groups each other, significant differences were found between groups of 4, 5 and 6 (Figure 3). Teppola et. al. found long neurites when they treated by combinations of retinoic acid and cholesterol, similarly to our results [6].

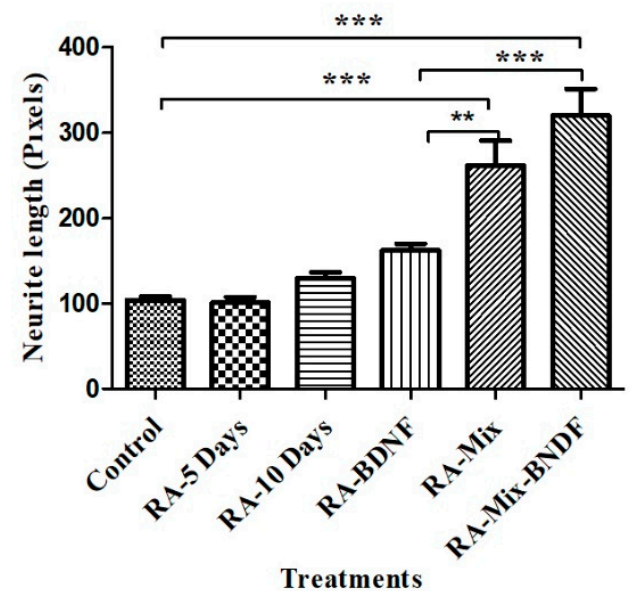

Figure 3. Neurite lengths of differentiated and undifferentiated SH-SY5Y cells. * Statistically significant changes $\left({ }^{* *} p<0.05\right.$ RA-BDNF vs RA-Mix, ${ }^{* * *} p<0.001$ control vs treated groups).

\section{Conclusions}

SH-SY5Y cells are frequently used cells in neuroscience related research. To reflect neuron-like phenotype or behavior, most of the researchers prefer to differentiate of the cells. However, ingredients of mediums and treatment period used in differentiation have been still controversial. In this report we examined the effects of treatment period and medium ingredients on differentiation 
levels of SH-SY5Y cells. We found that using of RA as pre-treatment of SH-SY5Y cells for 5 days and followed with BNDF, B27, Glutamax, dc-AMP, KCI, Neurobasal addition to the medium provides the best neuronal differentiation in the groups. We suggest that this medium can be used in the neuroscience related in-vitro studies to obtain more accurate results.

Acknowledgments: This research was supported in part by a grant (2017.KB.SAG.004) from Dokuz Eylul University Scientific Research Project Unit. We also would like to express our gratitude to Professor Kemal S. Korkmaz for guidance in immunofluorescence assays.

\section{References}

1. Biedler, J.L.; Helson, L.; Spengler, B.A. Morphology and growth, tumorigenicity, and cytogenetics of human neuroblastoma cells in continuous culture. Cancer Res. 1993, 33, 2643-2652.

2. Encinas, M.; Iglesias, M.; Liu, Y.; Wang, H.; Muhaisen, A.; Ceña, V.; Gallego, C.; Comella, J.X. Sequential treatment of SH-SY5Y cells with retinoic acid and brain-derived neurotrophic factor gives rise to fully differentiated, neurotrophic factor-dependent, human neuron-like cells. J. Neurochem. 2000, 75, 991-1003.

3. Kovalevich, J.; Langford, D. Considerations for the use of SH-SY5Y neuroblastoma cells in neurobiology. Methods Mol. Biol. 2013, 1078, 9-21.

4. Cheung, Y.T.; Lau, W.K.; Yu, M.S.; Lai, C.S.; Yeung, S.C.; So, K.F.; Chang, R.C. Effects of all-trans-retinoic acid on human SH-SY5Y neuroblastoma as in vitro model in neurotoxicity research. Neurotoxicology 2009, 30, 127-135.

5. Shipley, M.M.; Mangold, C.A.; Szpara, M.L. Differentiation of the SH-SY5Y human neuroblastoma cell line. J. Vis. Exp. 2016, 17, 53193.

6. Teppola, H.; Sarkanen, J.R.; Jalonen, T.O.; Linne, M.L. Morphological differentiation towards neuronal phenotype of sh-sy5y neuroblastoma cells by estradiol, retinoic acid and cholesterol. Neurochem. Res. 2016, $41,731-747$.

7. Agholme, L.; Lindström, T.; Kågedal, K.; Marcusson, J.; Hallbeck, M. An in vitro model for neuroscience: Differentiation of SH-SY5Y cells into cells with morphological and biochemical characteristics of mature neurons. J. Alzheimers Dis. 2010, 20, 1069-1082.

(C) 2018 by the authors. Licensee MDPI, Basel, Switzerland. This article is an open access article distributed under the terms and conditions of the Creative Commons Attribution (CC BY) license (http://creativecommons.org/licenses/by/4.0/). 